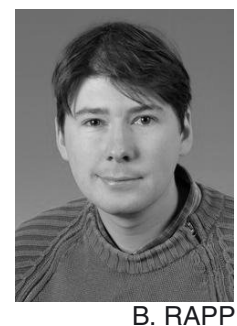

\title{
Développement d'un calorimètre à eau pour la dosimétrie au LNE-LNHB
}

\section{Development of a water calorimeter for dosimetry at $\mathrm{LNE}-\mathrm{LNHB}$}

\author{
Benjamin RAPP, Aimé OSTROWSKY et Josiane DAURES
}

CEA, LIST, Laboratoire national Henri Becquerel (LNE-LNHB), 91191 Gif-sur-Yvette Cedex, France, benjamin.rapp@cea.fr

\section{Résumé}

La calorimétrie est la meilleure technique disponible pour mesurer de façon absolue la dose absorbée. Les calorimètres à eau et en graphite sont utilisés comme référence dans la plupart des laboratoires de métrologie des rayonnements ionisants. Le LNE-LNHB dispose d'un savoir-faire reconnu dans la fabrication et la mise en œuvre des calorimètres en graphite et équivalent tissu. Le calorimètre en graphite est aujourd'hui le dosimètre de référence pour les faisceaux de photons et électrons au LNE-LNHB. Associé à une procédure de transfert du graphite vers l'eau, il mène à la référence de dose absorbée dans l'eau qui est la grandeur de référence en radiothérapie. Un calorimètre à eau a été récemment développé au LNE-LNHB afin de diversifier les méthodes permettant d'établir une référence en termes de dose absorbée dans l'eau.

MOTS CLÉS : MÉTROLOGIE DES RAYONNEMENTS IONISANTS, CALORIMÉTRIE, DOSE ABSORBÉE, RADIOTHÉRAPIE.

\footnotetext{
Abstract

Calorimetry is the best technique available to perform absolute measurement of absorbed dose. Graphite or water calorimeters are mainly used as reference for absorbed dose in water in most of the national metrology laboratories involved in ionizing radiations. $L N E-L N H B$ has a long experience with graphite and tissue-equivalent calorimeters. Graphite calorimeter is nowadays the reference dosimeter for photon and electron beams at LNE-LNHB. Associated with a transfer procedure from graphite to water, it leads to the reference of absorbed dose to water which is the reference quantity for radiotherapy. A water calorimeter is being developed currently in order to compare different ways to establish the standards of absorbed dose to water at $L N E-L N H B$.
}

\section{Introduction}

La calorimétrie dans l'eau est développée au LNHB en complément de la calorimétrie dans le graphite $[1,2]$ afin de disposer de plusieurs voies indépendantes pour mesurer de façon absolue la dose absorbée dans l'eau. Avec cette méthode, contrairement à la calorimétrie dans le graphite, la dose absorbée dans l'eau est mesurée directement dans le milieu de référence en radiothérapie, domaine des rayonnements ionisants le plus exigeant en terme d'incertitude. On s'affranchit ainsi de la procédure de transfert de la dose absorbée dans le graphite vers la dose absorbée dans l'eau.

Néanmoins la calorimétrie dans l'eau pose des problèmes spécifiques [3] tels que la maîtrise des mouvements de convection, la maîtrise de la pureté et du contenu en gaz dissous de l'eau, et la mesure de l'élévation de température qui est environ six fois plus faible (environ $240 \mu \mathrm{K} \cdot \mathrm{Gy}^{-1}$ ) qu'en calorimétrie dans le graphite pour une même dose.

\section{Principe de la calorimétrie dans l'eau}

La dose absorbée dans l'eau $(D)$ est définie avec la relation (1) :

$$
\begin{aligned}
D & =\frac{E}{m}=\frac{Q \cdot \prod_{i} k_{i}}{m} \\
& =\frac{m \cdot C_{p} \cdot \Delta T \cdot \prod_{i} k_{i}}{m}=C_{p} \cdot \Delta T \cdot \prod_{i} k_{i},
\end{aligned}
$$


où $E$ est l'énergie communiquée à la matière par les rayonnements ionisants, $m$ est la masse de matière de l'élément de volume considéré, $C_{p}$ la capacité calorifique de l'eau, $\Delta T$ l'élévation de la température mesurée, et les $k_{i}$ les différents facteurs correctifs qui seront détaillés dans le paragraphe 4 .

On remarque que la masse s'élimine dans le rapport. La mesure de la quantité de chaleur $Q$ qui apparaît lors de l'irradiation dans la masse de matière du volume sensible $m$ est un accès direct à la dose absorbée puisqu'il ne fait pas appel à d'autres phénomènes physiques qui conduisent à la grandeur mesurée, tels que l'énergie nécessaire pour créer une paire d'ions dans les chambres d'ionisation ou le rendement radiochimique pour le dosimètre chimique au sulfate ferreux (dosimètre dit de Fricke).

La faible diffusivité thermique de l'eau $(1,3 \times$ $10^{-7} \mathrm{~m} \cdot \mathrm{s}^{-1}$ par rapport à la valeur pour le graphite qui est de $9,1 \times 10^{-5} \mathrm{~m} \cdot \mathrm{s}^{-1}$ ) est mise à profit pour limiter les transferts thermiques. Dans certaines conditions, l'inertie thermique de l'eau permet ainsi de réaliser une mesure de température ponctuelle. Si les gradients de dose absorbée, donc de température, sont modérés, alors les transferts thermiques, proportionnels aux gradients de température, sont faibles sur la durée de la mesure et font l'objet d'estimations expérimentales et calculées.

\section{Description du calorimètre}

\subsection{Enceinte thermique}

Le calorimètre est constitué d'un «fantôme » de référence en eau entouré d'une enceinte thermique. Le fantôme est une cuve en PMMA (polyméthacrylate de méthyle) avec une épaisseur de paroi de $15 \mathrm{~mm}$ et de dimensions externes $30 \mathrm{~cm} \times 30 \mathrm{~cm} \times 30 \mathrm{~cm}$. La fenêtre d'entrée face au faisceau est amincie à $4 \mathrm{~mm}$ sur une surface rectangulaire de $12 \mathrm{~cm} \times 12 \mathrm{~cm}$. La cuve de référence constitue la partie interne d'une cuve à double paroi dans laquelle circule un fluide réfrigérant (sauf au niveau de la fenêtre d'entrée) permettant de maintenir la température à $4{ }^{\circ} \mathrm{C}$ (correspondant au maximum de densité de l'eau pour s'affranchir au maximum des mouvements de convection dans le fantôme). Pour limiter les perturbations induites par les variations de température de la salle d'irradiation, cette double cuve est entourée d'une épaisseur de $8 \mathrm{~cm}$ de polystyrène expansé. L'ensemble (Figs. 1 et 2) est recouvert d'une coque rigide de protection en PVC (polychlorure de vinyle).

La cuve est pourvue d'un agitateur magnétique permettant d'homogénéiser l'eau à l'intérieur, il est principalement utilisé pour accélérer la descente en température de l'eau jusqu'à sa valeur de consigne de $4{ }^{\circ} \mathrm{C}$.

Un dispositif supplémentaire permet de maintenir une couche d'air froid à $4{ }^{\circ} \mathrm{C}$ au niveau de la fenêtre d'entrée. Ainsi, seule une plaque de polystyrène d'épaisseur $2 \mathrm{~cm}$ et quatre feuilles de Mylar d'épaisseur 0,1 mm sont

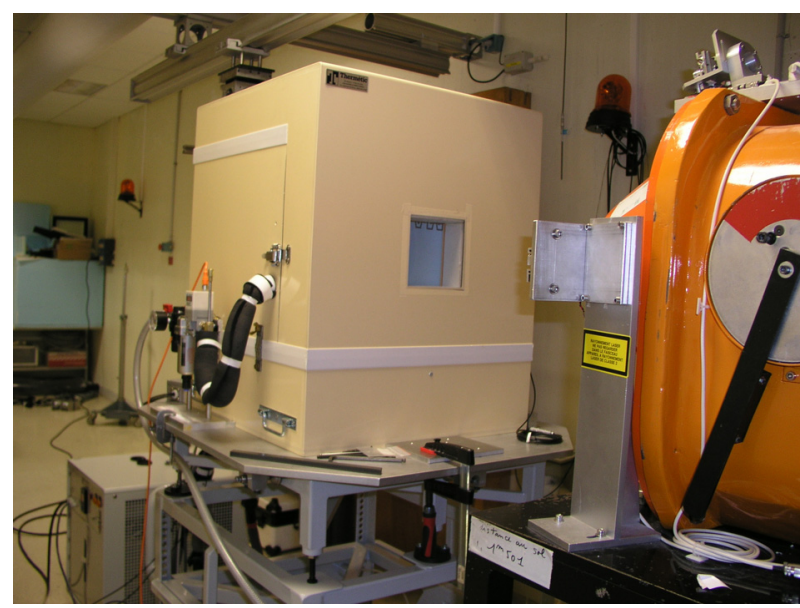

Fig. 1. - Calorimètre à eau positionné face à la source de cobalt-60 n $2 \mathrm{C}$ du LNHB-LNE.

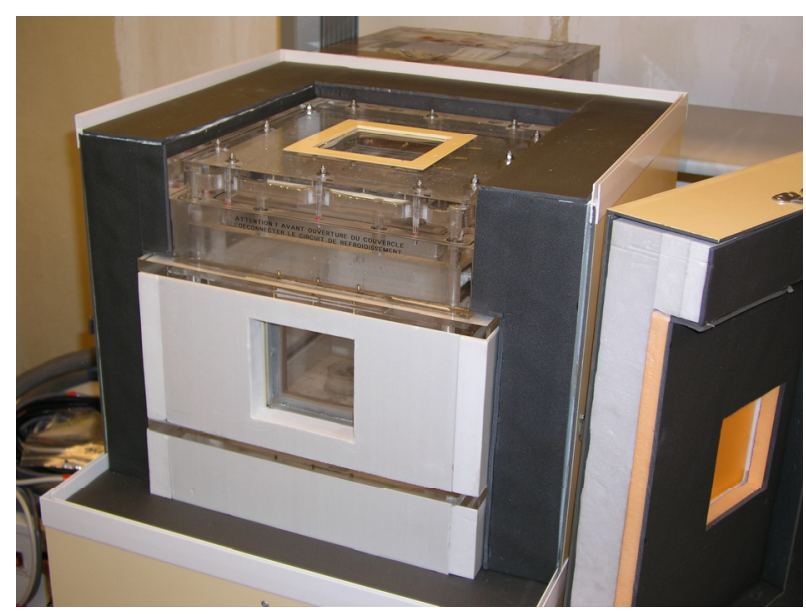

Fig. 2. - Cuve en PMMA à double paroi du calorimètre eau.

interposés sur le trajet du faisceau entre la source et la face avant du fantôme (cuve interne).

\subsection{Ampoule en quartz et eau pure}

La mesure de l'élévation de la température ne peut être directement faite dans l'eau de la cuve du calorimètre. La mesure de la température est réalisée à l'intérieur d'une ampoule cylindrique en quartz, qui permet d'isoler un volume d'eau dont la pureté et la saturation en gaz dissous peut être contrôlée. L'ampoule joue en plus le rôle de barrière de convection et vient ralentir, dans une certaine mesure, la diffusion thermique sous irradiation. Le quartz a été utilisé pour réduire la contamination de l'eau par des impuretés. Les faces parallèles de l'ampoule sont constituées de feuilles de quartz amincies d'épaisseur $0,8 \mathrm{~mm}$. Le diamètre de l'ampoule est de $10 \mathrm{~cm}$.

Deux sondes de température peuvent être disposées en même temps dans l'ampoule, de façon symétrique au centre géométrique.

L'ampoule est fixée sur un support en PMMA qui permet de la maintenir et de la positionner selon trois axes. 


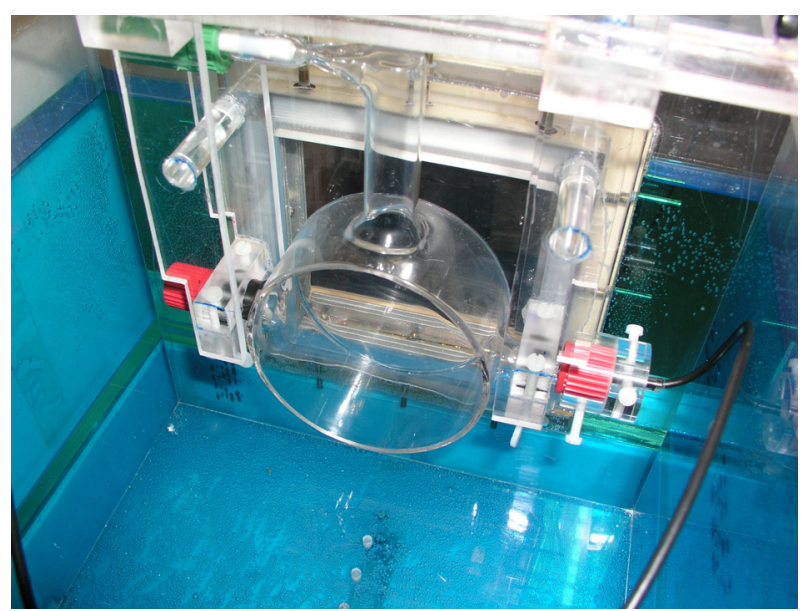

Fig. 3. - Ampoule en quartz permettant d'isoler un volume d'eau ultra-pure positionnée à l'intérieur de la cuve du calorimètre.

Le support repose lui-même sur deux rails en PMMA collés à l'intérieur du fantôme (Fig. 3).

Pour que la mesure de la dose dans l'eau soit fiable, il faut disposer de l'eau la plus pure possible et sans oxygène dissous, ceci afin que toute l'énergie communiquée à l'eau par les photons soit transformée en chaleur. On parle alors de défaut de chaleur $h$ de l'eau nul. Pour produire de l'eau ultra-pure un appareil de filtration MILLIPORE MILLIQ A10 est utilisé. Il est alimenté avec de l'eau bi-distillée produite au laboratoire. L'eau produite n'est pas stockée, elle est utilisée immédiatement pour remplir l'ampoule de saturation avant usage. Pour enlever l'oxygène dissous, on sature l'eau dans une ampoule en quartz en faisant buller de l'azote de haute pureté.

\subsection{Sonde de température}

La sonde de température doit perturber le moins possible les mesures des points de vue dosimétrique et thermique. Étant située à l'intérieur de l'ampoule, elle doit être étanche et ne pas être une source de contamination chimique de l'eau pure utilisée pour les mesures. Pour minimiser le plus possible ces effets, la sonde est réalisée avec un capillaire en quartz, dont le diamètre externe est de $0,6 \mathrm{~mm}$.

La mesure de température est effectuée par une thermistance à coefficient de température négatif (CTN) placée à l'intérieur du capillaire (Fig. 4). Les thermistances utilisées ont une grande sensibilité $(4 \% / \mathrm{K})$ et un faible volume. Leur composition à base d'oxydes métalliques et leur enrobage de verre leur assure une très bonne résistance aux irradiations.

Les sondes de température sont étalonnées avant chaque campagne de mesures. La sonde à étalonner est plongée dans un bain régulé en température (stabilité : $5 \mathrm{mK}$ et uniformité : $7 \mathrm{mK}$ ) en même temps qu'une sonde de référence Pt-100 étalonnée au LNE.

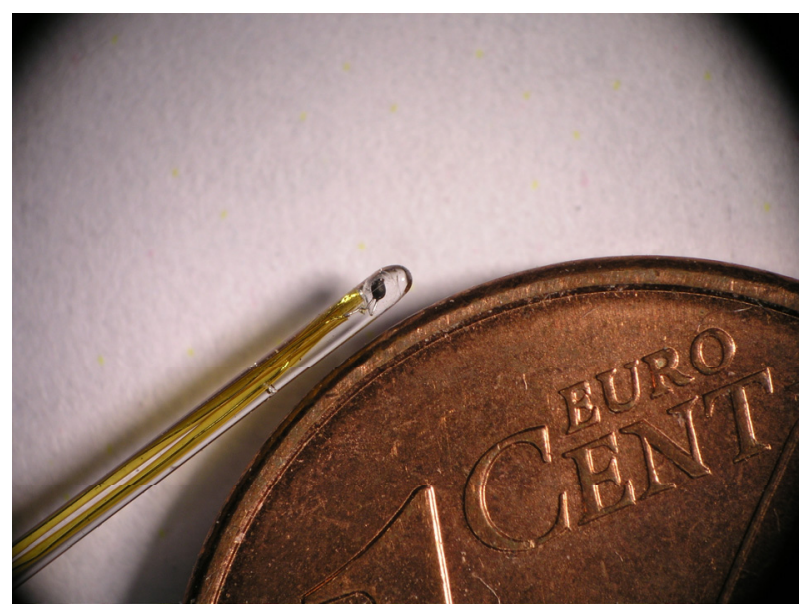

Fig. 4. - Extrémité d'une sonde de température en quartz.

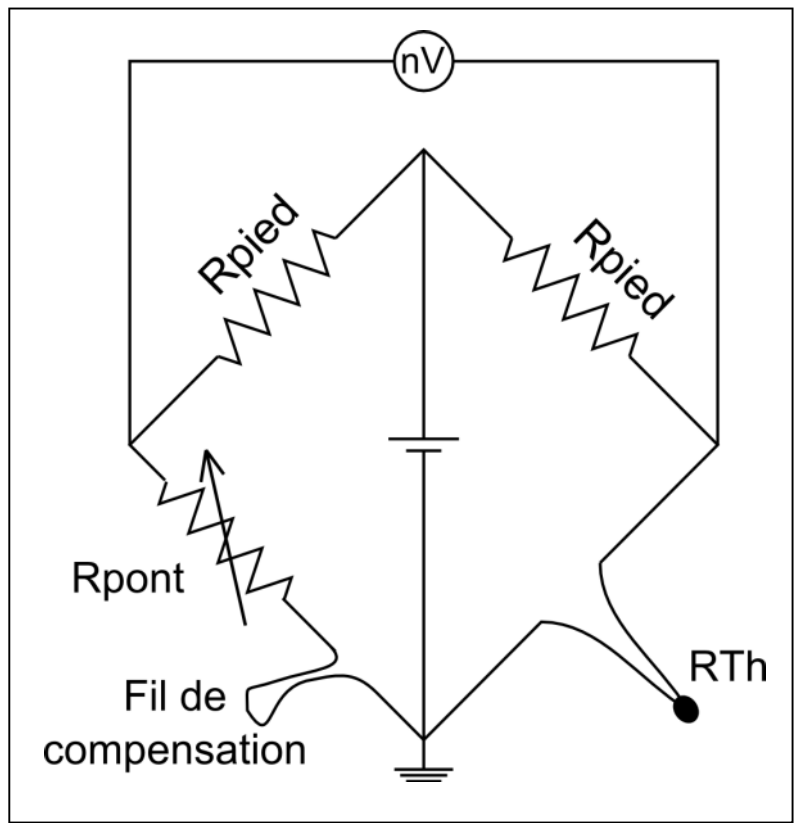

Fig. 5. - Schéma du pont de Wheatstone utilisé pour mesurer la résistance de la thermistance $\left(R_{\mathrm{Th}}\right)$ de la sonde de température.

\section{4. Électronique}

La mesure de la résistance des thermistances se fait par deux ponts de Wheatstone utilisés en courant continu (voir schéma Fig. 5). La tension d'alimentation des ponts est variable de $0,2 \mathrm{~V}$ à $2,5 \mathrm{~V}$, les résistances de pied sont des résistances de précision VISHAY de $8000 \Omega$. Les sondes de température sont câblées en quatre fils avec un fil de compensation relié à la branche opposée à la thermistance dans le pont de Wheatstone. Pour équilibrer approximativement le pont, il est utilisé soit une boite à décade, soit un jeu fixe de résistances VISHAY dont la valeur est adaptée pour chaque série de thermistances, de façon à se placer dans la gamme la plus petite du nanovoltmètre.

Comme le pont n'est jamais parfaitement équilibré, on utilise l'équation (2) pour calculer la résistance de la 
thermistance $R_{\text {Th }}$ qui varie faiblement autour de sa valeur nominale à $4{ }^{\circ} \mathrm{C}$ :

$$
R_{\mathrm{Th}}=\frac{R_{\text {pied }}\left(E \times R_{\text {pont }}-V \times R_{\text {pont }}-V \times R_{\text {pied }}\right)}{V \times R_{\text {pont }}+V \times R_{\text {pied }}+E \times R_{\text {pied }}},
$$

où $E$ est la tension d'alimentation du pont et $V$ la tension mesurée par le nanovoltmètre.

\section{Mesures et facteurs de correction}

La dose absorbée dans l'eau est calculée à partir de l'élévation de température par l'équation (3) :

$$
D_{W}=C_{p} \cdot \Delta T \cdot(1-h)^{-1} \cdot k_{c} \cdot k_{p} \cdot k_{\rho},
$$

où $C_{p}$ est la capacité calorifique de l'eau à pression constante $\left(1,013 \times 10^{5} \mathrm{~Pa}\right)$ et à une température de $4{ }^{\circ} \mathrm{C}$, $\Delta T$ est l'élévation de température mesurée sous irradiation et $h$ est le défaut de chaleur de l'eau. La mesure est corrigée des effets de conduction thermique $\left(k_{c}\right)$, de la perturbation dosimétrique apportée par l'ampoule et l'enceinte du calorimètre $\left(k_{p}\right)$ et de la différence de densité de l'eau $\left(k_{\rho}\right)$ entre $4{ }^{\circ} \mathrm{C}$ et $20{ }^{\circ} \mathrm{C}$. Une correction sur le positionnement de la sonde de température, en termes de centrage sur l'axe du faisceau, de distance et de profondeur dans l'eau sont également effectués, si nécessaire, suivant les conditions expérimentales de mesure.

\subsection{Mesure de la température}

La faible diffusivité thermique de l'eau permet de limiter les transferts thermiques, il est ainsi possible de réaliser une succession d'irradiations de quelques minutes avec un temps de repos de l'ordre de l'heure permettant aux températures de s'homogénéiser dans la cuve du calorimètre. Pour déterminer l'élévation de la température $\Delta T$ sous irradiation, on effectue un ajustement linéaire de la température avant et après irradiation sur un intervalle de temps égal au temps d'irradiation ( $\left.t_{\text {irr. }}\right)$, tel qu'explicité sur la figure 6. L'incertitude sur la valeur de $\Delta T$ mesurée est estimée à partir de la dispersion statistique des résultats, qui incluent l'incertitude de la méthode d'extrapolation en plus de la stabilité thermique de l'enceinte (dérive thermique inférieure à $20 \mu \mathrm{K} \cdot \mathrm{min}^{-1}$ ) et de l'influence des bruits électriques et thermiques sur la sonde et l'électronique de mesure (bruit d'environ $30 \mu \mathrm{K})$.

\subsection{Calcul du facteur de correction de la conduction thermique}

Les mesures calorimétriques nécessitent d'être corrigées des effets thermiques susceptibles d'influencer la mesure de l'élévation de la température due à l'interaction des rayonnements ionisants seuls. En effet, les transferts de chaleur par conduction, convection et rayonnement peuvent fausser la mesure de température. La conductivité thermique de l'eau est faible comparée à celle du graphite ou des métaux, il est donc possible de faire une mesure «locale » d'élévation de température induite par une irradiation de quelques minutes, avant que

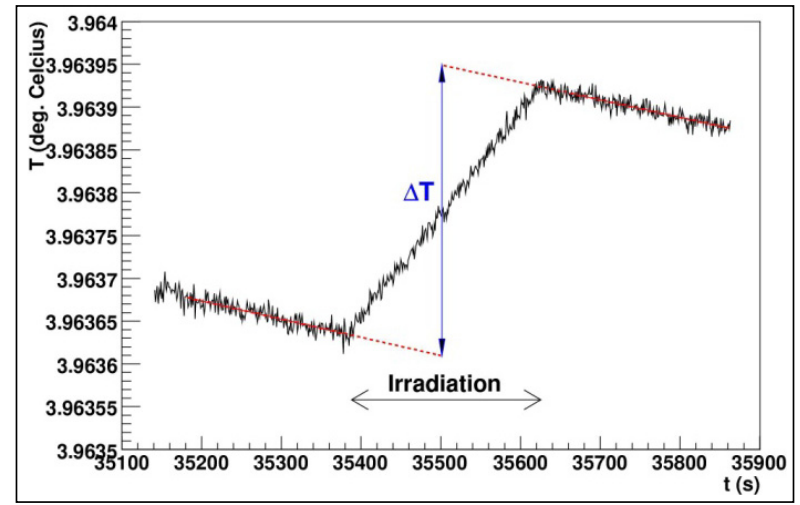

Fig. 6. - Exemple de mesure de l'élévation de température pendant une irradiation de $4 \mathrm{~min}$. La détermination de la valeur de $\Delta T$ est réalisée en extrapolant à mi-irradiation les ajustements linéaires sur les périodes pré et post-irradiation.

la chaleur déposée en un point ne diffuse dans le volume d'eau. En opérant à une température de $4{ }^{\circ} \mathrm{C}$ au maximum de densité de l'eau, et en utilisant une ampoule de confinement, on minimise l'effet de la convection naturelle dans le volume d'eau. Par contre, à cause de cette ampoule de confinement et de la présence inévitable de la sonde de température, le milieu est inhomogène, d'autant plus que le matériau utilisé, du quartz pour préserver la pureté de l'eau, possède une capacité calorifique très différente de l'eau. Pour une même dose absorbée, l'élévation de température dans le quartz est environ six fois plus importante que dans l'eau. Il est donc essentiel de simuler les échanges thermiques par conduction (dans le calorimètre au niveau de l'ampoule) se produisant pendant les mesures, y compris les périodes de stabilisation avant et après l'irradiation. Le facteur correctif associé, permettant de corriger l'élévation de la température de l'eau est appelé facteur de correction de la conduction thermique $k_{c}$.

Le code de calcul Monte-Carlo MCNPX [4] a permis de simuler la distribution du dépôt de dose en 3D à l'intérieur de la cuve du calorimètre, ampoule en quartz comprise. Le logiciel de calcul par éléments finis COMSOL [5], a ensuite été utilisé pour simuler les transferts thermiques par conduction au cours du temps, pendant et après l'irradiation, en utilisant comme source de chaleur externe la distribution d'énergie déposée calculée par Monte-Carlo. Le maillage utilisé est visible sur la figure 7, et l'élévation de température à un instant $t$ est montrée sur la figure 8.

En comparant l'élévation de la température obtenue par le calcul par éléments finis avec l'élévation de la température attendue en l'absence de transferts thermiques, on en déduit le facteur de correction de la conduction thermique à appliquer à chaque irradiation. Le facteur de correction varie en fonction de l'historique des irradiations, un exemple est visible sur la figure 9 pour quatre irradiations successives où les transferts thermiques à l'issue de chaque irradiation sont corrélés avec les irradiations antérieures. 


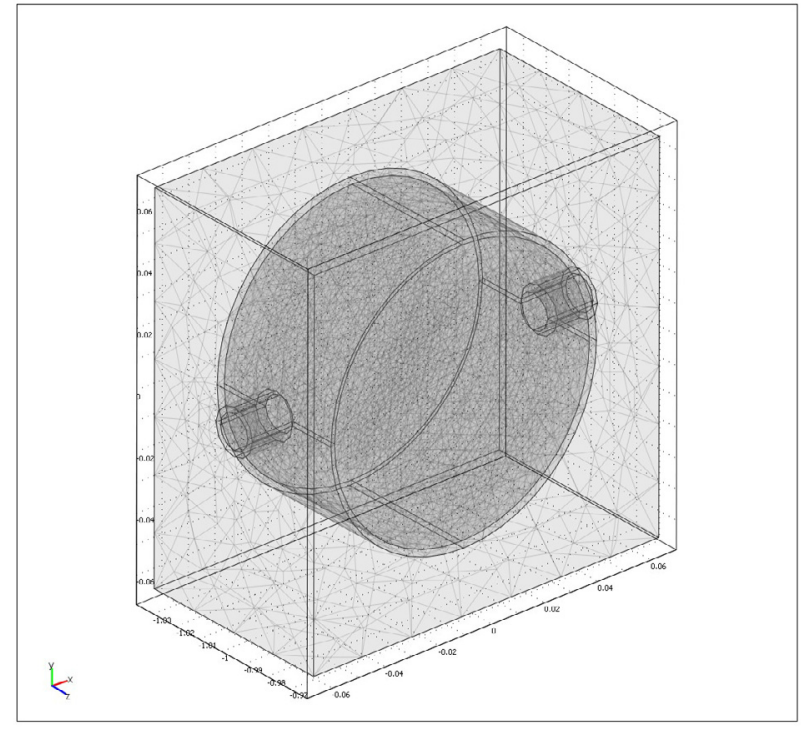

Fig. 7. - Maillage de l'ampoule en quartz utilisé dans le logiciel de calcul aux éléments finis COMSOL.

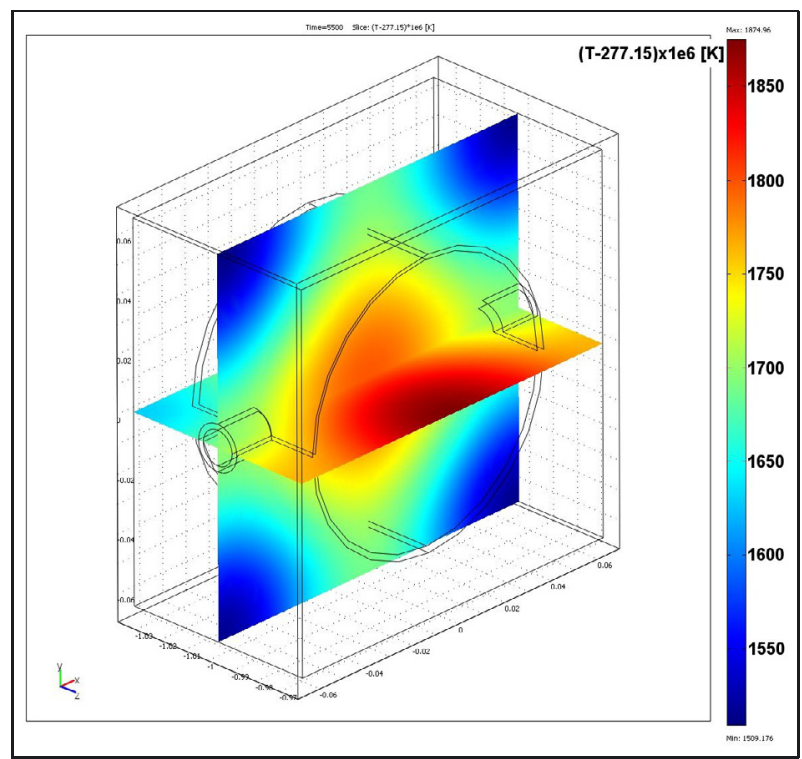

Fig. 8. - Élévation de température à un instant $t$ sous irradiation calculée avec le logiciel de calcul par éléments finis COMSOL.

\subsection{Facteur de perturbation dosimétrique}

Les mesures effectuées avec le calorimètre eau nécessitent d'être corrigées des perturbations dosimétriques inhérentes aux différences entre le calorimètre et une cuve de radiothérapie classique. Ceci afin de pouvoir comparer la mesure de la dose absorbée dans l'eau du calorimètre avec la dose absorbée de référence mesurée par d'autres méthodes primaires. Les perturbations dosimétriques sont principalement le fait de l'enceinte thermique du calorimètre et de l'ampoule en quartz qui contient la sonde de température. Ce facteur de correction appelé $k_{p}$ a été déterminé par des mesures relatives avec une chambre d'ionisation placée successivement dans une cuve de radiothérapie et dans la cuve du calorimètre

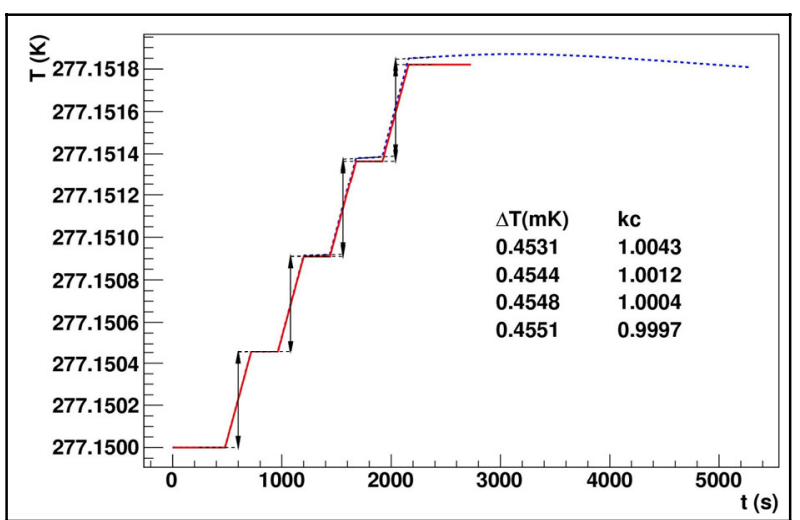

Fig. 9. - Élévation de la température au cours de quatre irradiations successives simulée avec COMSOL (ligne en pointillés) et attendue en l'absence de transferts thermiques (ligne pleine).

à la même profondeur, et la même distance de la source de cobalt-60 du LNE-LNHB. Ce facteur de correction est également calculé, pour vérification, au moyen de la méthode Monte-Carlo en incluant une géométrie détaillée du calorimètre.

\subsection{Défaut de chaleur de l'eau}

Le défaut de chaleur de l'eau est défini par la relation (4) :

$$
h=\frac{E_{a}-E_{h}}{E_{a}},
$$

avec $E_{a}$ l'énergie absorbée par le milieu, c'est-à-dire l'énergie déposée par le rayonnement, et $E_{h}$ l'énergie apparaissant sous forme de chaleur.

Ce bilan n'est pas nul si l'énergie apportée par les rayonnements ionisants est incomplètement convertie en chaleur. En effet les rayonnements ionisants sont à l'origine du processus de radiolyse de l'eau qui initie un ensemble complexe de réactions chimiques dont le bilan thermique final peut être exothermique ( $h$ négatif) ou endothermique ( $h$ positif). La valeur du défaut de chaleur dépend fortement du contenu en oxygène dissous et en impuretés organiques de l'eau utilisée. Le défaut de chaleur théorique est nul pour de l'eau ultra pure saturée avec un gaz inerte vis-à-vis des réactions de radiolyse, tel l'azote.

\section{Mesures dans un faisceau de photons $\gamma$ du LNE-LNHB}

Des mesures du débit de dose absorbée dans l'eau ont été réalisées dans le faisceau de cobalt- $60 \mathrm{n}^{\circ} 2 \mathrm{C}$ de référence du LNE-LNHB. Le bilan d'incertitude de mesure est présenté dans le tableau 1. Les incertitudes de type A et type B sont données pour les différentes sources d'incertitudes prises en compte, qui sont l'incertitude sur l'étalonnage et le positionnement de la sonde de température, l'incertitude sur la valeur de la capacité calorifique de l'eau utilisée dans le calcul du débit de dose absorbée 
Tableau 1

Bilan d'incertitude.

\begin{tabular}{|l|c|c|c|}
\hline & \multirow{2}{*}{ Source d'incertitude } & Valeur & \multicolumn{2}{|c|}{ Incertitude relative (\%) } \\
\cline { 3 - 4 } & - & 0,10 & \\
\hline Étalonnage de la sonde de température & - & & 0,30 \\
\hline Positionnement de la sonde de température & 4204,8 & & 0,10 \\
\hline Capacité calorifique de l'eau $\left(\mathrm{J}^{\prime} \mathrm{kg}^{-1} \cdot \mathrm{K}^{-1}\right)$ & $*$ & & 0,10 \\
\hline Correction de la conduction thermique $k_{c}$ & 1,0032 & 0.15 & \\
\hline Correction de la perturbation dosimétrique $k_{p}$ & 0 & & 0,30 \\
\hline Défaut de chaleur de l'eau $h$ & 1,00032 & 0,01 & \\
\hline Correction de la densité de l'eau $k_{\rho}$ & - & 0,07 & \\
\hline Reproductibilité de la mesure de $\Delta T(N=420)$ & & 0,19 & 0,45 \\
\hline Somme quadratique & & \multicolumn{2}{|c|}{0,49} \\
\hline Incertitude type relative sur le débit de dose dans l'eau $D_{w}$ & & \\
\hline
\end{tabular}

* Facteur de correction de la conduction thermique pour un temps d'irradiation $t_{\text {irr. }}$ de $240 \mathrm{~s}$, dont la valeur est différente pour chacun des quatre paliers d'irradiation successifs (voir Fig. 9) $: k_{c}=(1,0043 ; 1,0012 ; 1,0004 ; 0,999$ 7).

à partir de l'élévation de température, l'incertitude sur les facteurs de correction de la conduction thermique $\left(k_{c}\right)$ et de la perturbation dosimétrique $\left(k_{p}\right)$ discutés dans la section précédente. L'incertitude sur le défaut de chaleur $h$ a été estimée à $0,3 \%$ à partir d'une simulation numérique des réactions de radiolyse dans l'eau basée sur des mesures expérimentales du contenu en oxygène dissous et en impuretés organiques. L'incertitude sur le facteur de correction pour la différence de densité de l'eau $\left(k_{\rho}\right)$ entre $4{ }^{\circ} \mathrm{C}$ et $20{ }^{\circ} \mathrm{C}$ est également incluse. Les deux principales sources d'incertitudes sont ainsi le positionnement de la sonde de température et le défaut de chaleur de l'eau. La dispersion des élévations de la température mesurées $(\Delta T)$ est importante (environ 1,2\%) mais la réalisation d'un grand nombre de mesures (ici une série de 420 mesures) permet de réduire l'incertitude statistique sur la valeur moyenne à $0,07 \%$. Ce qui est justifié du fait du caractère aléatoire de cette dispersion, principalement due aux bruits thermique et électrique associés aux sondes de température.

\section{Conclusion}

Le calorimètre à eau du LNE-LNHB est maintenant opérationnel et les premières mesures de dose face à une source de ${ }^{60} \mathrm{Co}$ ont été effectuées. Les résultats ont montré qu'il était possible de mesurer un débit de dose absorbée dans l'eau de l'ordre de la dizaine de grays par heure avec une incertitude-type de $0,5 \%$. L'incertitude obtenue étant comparable avec celle sur la référence actuelle $(0,46 \%)$ basée sur la calorimétrie dans le graphite. Le calorimètre à eau permet maintenant de disposer d'une nouvelle méthode de mesure de la dose absorbée dans l'eau au LNE-LNHB totalement indépendante de celle utilisée jusqu'à présent, c'est-à-dire la mesure de la dose dans le graphite obtenue par calorimétrie, suivie d'un transfert graphite-eau. Ce nouvel instrument sera ensuite utilisé pour caractériser différents faisceaux primaires du laboratoire comme les faisceaux de photons et d'électrons de haute énergie et les rayons X de moyenne énergie.

\section{Références}

[1] Daures J. et OSTROWSKY A., "New constanttemperature operating mode for graphite calorimeter at LNE-LNHB”, Phys. Med. Biol., 50, 2005, 4035.

[2] SeuntJens J. et DuAne S., "Photon absorbed dose standards", Metrologia, 46, 2009, S39-S58.

[3] Ross C.K. et KLASSEN N.V., "Water calorimetry for radiation dosimetry", Phys. Med. Biol., 41, 1996, 1-29.

[4] MCNPX - Monte Carlo N-Particle Transport Code System for Multiparticle and High Energy Applications, http://monpx.lanl.gov/.

[5] COMSOL 3.4 Multiphysics, http://www.comsol.com/. 